Rebollo-Quintela, N. \& Losada-Puente, L. (2021). Modelo competencial del maestro en educación infantil. Revista Electrónica Interuniversitaria de Formación del Profesorado, 24(3), 115-132.

DOI: https://doi.org/10.6018/reifop.457881

\title{
Modelo competencial del maestro en educación infantil
}

\author{
Nuria Rebollo-Quintela, Luisa Losada-Puente \\ Universidade da Coruña
}

\section{Resumen}

La formación inicial y permanente del maestro en Educación Infantil debe ajustarse a los retos educativos actuales respondiendo a las recomendaciones de la Unión Europea en materia competencial y a la necesidad de un modelo global docente. Partiendo de estas premisas se propuso un Modelo para definir el perfil del maestro en cuatro dimensiones competenciales: educador y guía, miembro de una organización, interlocutor y referente, e investigador e innovador. El objetivo general del presente estudio fue conocer la relevancia que el futuro profesorado otorga a las mismas para su ejercicio profesional. Se empleó una metodología cuantitativa, descriptivo-exploratorio, mediante la aplicación de un cuestionario a 144 estudiantes de $3^{\circ}$ y $4^{\circ}$ de Grado en Educación Infantil. Las cuatro competencias fueron consideradas altamente relevantes, destacando significativamente Interlocutor y referente $(p<.001$ ) y sus subcompetencias (gestión y promoción de valores y convivencia, compromiso personal y ético; y habilidades personales, sociales y relacionales). En conclusión, se puede extraer que el reconocimiento y valoración positiva hacia su formación inicial y, con ello, a disponer de un modelo de formación integral repercutirá, no solo en la capacitación adquirida, sino también en su predisposición hacia la búsqueda de mejora continua.

\section{Palabras clave}

Formación inicial; educación superior; maestros en formación; perfil profesional

\section{Contacto:}

Nuria Rebollo Quintela, nuria.rebollo@udc.es.Facultad de Ciencias de la Educación, Campus de Elviña, s/n. Universidade da Coruña, CP 15071, A Coruña. Tfno.: 881161816. 


\title{
Competencial model of the teacher in early education
}

\begin{abstract}
The initial and permanent training of the teacher in Early Childhood Education must be adapted to the current educational challenges in response to the recommendations of the European Union in terms of competency and to the need for a global teaching model. Based on this, a Model was proposed to define the teacher's profile based on four competency dimensions: educator and guide, member of an organization, interlocutor and referent, and researcher and innovator. The general objective of the present study was to know the relevance that future teachers give to them for their professional exercise. A quantitative methodology, descriptive-exploratory, was carried out by applying a questionnaire to 144 students from the 3rd and 4th grade of the Degree in Early Childhood Education. The four competencies were considered highly relevant, highlighting Interlocutor and referent $(p<$ .001) and its subcompetences (management and promotion of values and coexistence, personal and ethical commitment; and personal, social and relational skills). It can be concluded that the positive recognition and assessment toward their initial training and, therefore, to have a comprehensive training model will have an impact not only on the training acquired, but also on their predisposition towards the search for continuous improvement.
\end{abstract}

\section{Key words}

Initial training; higher education; teacher in training; professional profile

\section{Introducción}

Las numerosas transformaciones sociales, económicas, políticas y culturales vivenciadas en las últimas décadas han repercutido de forma directa sobre uno de los pilares fundamentales de las sociedades actuales: la educación. Si bien, en los diferentes niveles y etapas educativas se observan cambios en consonancia con la necesidad de adaptarse a las concepciones sociales sobre la equidad, la igualdad y la justicia social para ofrecer una respuesta adecuada a las necesidades y demandas en la formación inicial de maestros/as, esta metamorfosis dista mucho de realizarse en profundidad. Las escuelas actuales parecen no funcionar adecuadamente y ello puede venir motivado, entre otras razones, por una formación inadecuada o ineficiente del profesorado.

Este estudio plantea un análisis de la relevancia de las competencias adquiridas por las futuras y futuros maestros en Educación Infantil desde la perspectiva de los principales implicados en el proceso de enseñanza-aprendizaje en el Espacio Europeo de Educación Superior (EEES). Para ello, se formulan algunas cuestiones clave sobre el perfil competencial de esta titulación, que se enmarca en un contexto de cambio y adaptación a la realidad educativa actual en la que el docente debe dar respuesta haciendo uso del conjunto de habilidades, destrezas y actitudes adquiridas durante su formación inicial. 


\section{Perfiles competenciales en el contexto universitario}

El modelo actual de universidad es el resultado de un proceso de adaptación a los nuevos retos y demandas educativas procedentes de la sociedad (Pérez y Castaño, 2016) y que, a nivel internacional, se han resuelto a través de la modificación del diseño de los planes de estudios de las y los maestros, a través del desarrollo de competencias (Ramírez, 2015).

En España, la implantación del EEES ha supuesto no sólo un gran cambio en el proceso de enseñanza y en la nueva configuración de los aprendizajes en términos de competencias (Feisal, 2015; Gairín, 2011) sino en su función como institución social y como modelo de referencia en la transmisión de valores y principios éticos profesionales (Tejada y Ruíz, 2013). En el caso de la formación de maestras y maestros en Educación Infantil el desafío al que se enfrenta la universidad es doble. Por un lado, el derivado de su responsabilidad por crear ciudadanos comprometidos y críticos (Pérez y Castaño, 2016) y, por el otro, el de lograr que adquieran la capacitación que les permita enfrentarse a los retos del sistema educativo y se adapten a las necesidades de la escuela. Para lograr este fin, se propone un sistema de formación inicial en competencias que dota a los/as futuros/as profesionales de los conocimientos, estrategias y habilidades que les permita alcanzar el perfil profesional esperado y definido en el Libro Blanco del título de Grado en Magisterio (Agencia Nacional de Evaluación de la Calidad y Acreditación, ANECA, 2004) y en la Orden ECl/3854/2007, de 27 de diciembre, si bien parece que dichos planes se encuentran al margen de los ajustes y adaptaciones derivados de las demandas de la sociedad actual.

Se han realizado esfuerzos por configurar un mapa que refleje las competencias a dominar o, cuanto menos, a poseer por el docente (Gairín, 2011; Ramírez, 2015; Rosales López, 2013), dando voz a los discentes durante su formación inicial (Asensio Muñoz y Ruíz de Miguel, 2017; Domínguez, González, Medina y Medina, 2015; Muñoz, Rebollo y Espiñeira, 2014) y a profesionales en ejercicio (González-Fernández, Zabalza-Cerdeiriña, Medina-Domínguez y Medina-Rivilla, 2019). No obstante, es evidente que definir el perfil competencial de los/as Maestros/as en Educación Infantil no es tarea fácil si consideramos las exigencias y expectativas que se depositan en ellos. A su vez, la forma en que se valoran las actuaciones docentes y las expectativas de y hacia estos profesionales han cambiado (Cuadrado, Monroy y Montaño, 2011).

Se espera que el docente competente sea "capaz de enfrentarse y resolver una situaciónproblema movilizando de modo integrado una serie de recursos de los cuales dispone (cognitivos, sociales, afectivos, ...)" (Latorre, Aravena, Milos y García, 2010, p.289), siendo necesario un cambio en el rol y funciones, en su proceso de enseñanza-aprendizaje, en sus valores y en su modo de entender la educación. Todo ello se traduce en una formación universitaria que les capacite para enfrentarse a los retos del sistema educativo y adaptarse a diferentes contextos y necesidades de la escuela, al tiempo que esta responde a los cambios de la sociedad (Gil, Gómez y Rodríguez, 2013) y en una formación a lo largo de su vida, como resultado de someterse un proceso de redescubrimiento y crecimiento profesional (De Haro Rodríguez, Arnaiz-Sánchez y Núñez de Perdomo, 2020).

\section{Plan de formación del Maestro en Educación Infantil en Galicia}

De forma complementaria a lo establecido por la orden ECI/3854/2007, la Xunta de Galicia (Consellería de Cultura, Educación e Ordenación Universitaria, 2015), ha diseñado un Modelo Competencial, a partir del cual se definen las competencias y ámbitos de actuación del 
docente en Educación Infantil. Este modelo refleja la concepción de competencia que plantea Zabalza (citado en López, González y de León, 2014) como un constructo global integrado por conocimientos y habilidades que requieren de su desglose en unidades más específicas para cada función a desempeñar dentro de una tarea.

De forma más específica, dicho modelo clasifica las competencias genéricas de perfil por ámbitos de actuación (aula, centro y comunidad educativa) y en su aplicación a la realidad educativa actual para su transformación (cambio) y las circunscribe a una serie de indicadores (ver tabla 1).

Tabla 1

Modelo de Competencias da Rede de Formación de la Xunta de Galicia

\begin{tabular}{|c|c|c|c|}
\hline Ámbitos & Competencia & Subcompetencia & Descripción \\
\hline Aula & $\begin{array}{l}\text { Educador/a } \\
\text { guía en el } \\
\text { proceso de } \\
\text { aprendizaje y } \\
\text { desarrollo del } \\
\text { alumnado }\end{array}$ & $\begin{array}{l}\text { Programación, seguimiento y } \\
\text { evaluación } \\
\text { Didácticas específicas, metodologías, } \\
\text { TAC y alfin } \\
\text { Orientación pedagógica y atención a } \\
\text { la diversidad } \\
\text { Gestión de los espacios, recursos y } \\
\text { materiales de aprendizaje }\end{array}$ & $\begin{array}{l}\text { Emplea sus capacidades, } \\
\text { habilidades, conocimientos y } \\
\text { herramientas para generar y } \\
\text { motivar los procesos de } \\
\text { enseñanza del alumnado. }\end{array}$ \\
\hline Centro & $\begin{array}{l}\text { Miembro de } \\
\text { una } \\
\text { organización }\end{array}$ & $\begin{array}{l}\text { Acción tutorial y orientación } \\
\text { pedagógica, académica y profesional } \\
\text { Normativa } \\
\text { Organización, planificación y } \\
\text { coordinación } \\
\text { Gestión de la participación e } \\
\text { implicación en proyectos comunes } \\
\text { Técnicas de trabajo en grupo y } \\
\text { distribución de responsabilidades } \\
\text { individuales }\end{array}$ & $\begin{array}{l}\text { Desempeña funciones } \\
\text { relacionadas con la } \\
\text { organización, administración y } \\
\text { gestión del centro aplicadas a } \\
\text { los planes de trabajo. }\end{array}$ \\
\hline $\begin{array}{l}\text { Comunidad } \\
\text { Educativa }\end{array}$ & $\begin{array}{l}\text { Interlocutor/a } \\
\text { y referente en } \\
\text { la comunidad } \\
\text { educativa }\end{array}$ & $\begin{array}{l}\text { Habilidades personales, sociales y } \\
\text { relacionales } \\
\text { Gestión y promoción de valores y } \\
\text { convivencia, compromiso personal y } \\
\text { ético }\end{array}$ & $\begin{array}{l}\text { Desarrolla conocimientos, } \\
\text { valores, actitudes y } \\
\text { comportamientos que ayuden a } \\
\text { promover un ambiente positivo } \\
\text { en la escuela. }\end{array}$ \\
\hline
\end{tabular}

\begin{tabular}{lll}
\hline Cambio & $\begin{array}{l}\text { Investigador/a Investigación formativa, TAC y alfin } \\
\text { e innovador/a }\end{array}$ & $\begin{array}{l}\text { Aplica nuevas ideas, prácticas y } \\
\text { propuestas educativas. }\end{array}$
\end{tabular}

Nota: información extraída de Consellería de Cultura, Educación e Ordenación Universitaria (2017). “As competencias profesionais docentes. Modelo competencial da Rede de Formación". Galicia: Xunta de Galicia e CAFI.

El diseño de este modelo competencial se justifica por la necesidad de encaminar la oferta formativa de los maestros hacia un modelo más global e integrador, teniendo en cuenta la 
percepción que tienen estos agentes educativos. En consonancia con lo trabajado en estudios previos que analizan las concepciones del alumnado universitario respecto a su formación como maestros de educación infantil (Cuberos, Santamaría, Prados y Arias, 2019; Domínguez et al., 2015), de infantil y primaria (Asensio Muñoz y Ruíz de Miguel, 2017), de educación en general (Bas-Peña, Ferre-Jaén y Maurandi-López, 2020; Fernández, Hervás, Aparicio, Polo y Tallón, 2019) o de los docentes en activo (Consellería de Cultura, Educación e Ordenación Universitaria, 2017), el presente trabajo tiene por objeto conocer la relevancia que el/la maestro/a en formación inicial otorga a las competencias definidas en el Modelo de Competencias de la Xunta. Para alcanzar este objetivo general se delimitan varios objetivos específicos:

a) describir el grado de relevancia que otorga a los componentes del Modelo propuesto,

b) comprobar si existen diferencias estadísticamente significativas entre las competencias y subcompetencias en cuanto al grado de relevancia que le otorga, y

c) analizar las correlaciones existentes entre dichas competencias y subcompetencias.

\section{Metodología}

Se emplea una metodología cuantitativa, de tipo descriptivo-exploratorio, en consonancia con los trabajos desarrollados en los últimos años en la línea de análisis de la formación en competencias (Cejudo, Díaz, Losada y Pérez-González, 2016; González-Fernández et al., 2019; Martínez, 2018; Ramírez, 2015; etc.).

\section{Población y muestra}

La población sobre la que se ha realizado esta investigación fue el alumnado que cursa $3^{\circ}$ y $4^{\circ}$ del Grado de Educación Infantil de la Universidad de A Coruña. Los criterios de inclusión se basan en el cumplimiento de las siguientes condiciones: (a) estar matriculado en el Grado en Educación Infantil; (b) ser estudiante de $3^{\circ} \mathrm{O} 4^{\circ}$ curso; (c) pertenecer a la modalidad presencial en el momento en que se realizó la aplicación del instrumento, y (d) aceptar participar en el estudio.

Se ha empleado una muestra de 144 alumnos y alumnas, con 131 mujeres y 13 hombres. La edad de dicha muestra osciló entre 22 y 25 años $(M=22.5$; D.T. $=.29)$. U 58,7\% de la muestra $(\mathrm{n}=84)$, cursaban $3^{\circ}$ curso y un $41.3 \%(n=59) 4^{\circ}$ curso.

\section{Instrumento}

La recogida de datos se llevó a cabo a través del "Cuestionario de Perfiles Competenciales del Grado en Educación Infantil (CUPERCOM)". Se trata de un cuestionario autocumplimentado, con una escala tipo Likert de 6 puntos (1: nada y 6: mucho) donde el alumnado debía responder a su acuerdo o desacuerdo con respecto a la relevancia de las competencias presentadas; esto es, la importancia que le confiere a la formación inicial respecto a su futura práctica profesional. El instrumento fue sometido a una validación de contenido, para lo que se contó con la participación de seis jueces expertos, de los cuales tres pertenecieron al ámbito universitario (área de métodos de investigación y diagnóstico en 
educación, área de didáctica y organización escolar, y área de psicología evolutiva y de la educación) y tres docentes en ejercicio (diplomadas en magisterio en educación infantil). El análisis de la fiabilidad global del cuestionario indicó un valor excelente $(\alpha=.975)$.

\section{Procedimiento de obtención y análisis de los datos}

Los datos se recogieron en las aulas universitarias en las que se impartía docencia presencial en el segundo cuatrimestre. Con esta forma de aplicación, se logró una elevada tasa de participación.

El tratamiento cuantitativo de la información se realizó con apoyo del Paquete Estadístico IBM SPSS Statistics versión 25. Se comprobó el cumplimiento del supuesto de normalidad, a través de la prueba de bondad de ajuste de Kolmogorov-Smirnov $(\mathrm{p}<.001)$ y homogeneidad de las varianzas con la prueba de Levene ( $\mathrm{p}$ <.001 en su mayoría). Con ello, se determinó la necesidad emplear estadística no paramétrica; más concretamente, se utilizó Prueba de Friedman y Wilcoxon para muestras relacionadas, y la Rho de Spearman para identificar las correlaciones entre variables. Se estableció un nivel de confianza de 95\% ( $p<.05)$ para determinar la presencia de diferencias estadísticamente significativas.

\section{Resultados}

A continuación, se presentan los resultados del análisis descriptivo e inferencial sobre la relevancia que los/as futuros/as maestros/as otorgan a las competencias adquiridas durante su formación universitaria.

\subsection{Análisis de las competencias y subcompetencias del Modelo Competencial}

En primer lugar, el análisis descriptivo de las cuatro competencias del Modelo indicó que el alumnado otorga mayor relevancia a las competencias de comunidad educativa Interlocutor/a y referente $(\mathrm{Md}=6)$ y de centro Miembro de una organización $(\mathrm{Md}=5.75)$, frente a las de cambio Investigador/a e innovador/a $(M d=5.5)$ y de aula Educador y guía en el proceso de aprendizaje y desarrollo del alumnado $(\mathrm{Md}=5.5)$, siendo además estas dos las que presentaron una mayor variabilidad de sus respuestas.

La figura 1 refleja la representación gráfica de estas diferencias. Se aprecia que los valores de la mediana y percentiles fueron, en el caso de la competencia de comunidad educativa elevados, con mayor concentración de las respuestas de los sujetos, mientras que el valor de la mediana más bajo se situó en la competencia de cambio seguido por la competencia de aula, habiendo mayor variabilidad de las respuestas en la primera.

Teniendo en cuenta estos datos, se planteó la hipótesis sobre la existencia de diferencias estadísticamente significativas entre la relevancia que el alumnado otorgaba a cada competencia, confirmada a través de la prueba de Friedman $\left(X_{3}^{2}=15.70 ; p<.001\right)$. Posteriormente, la prueba de rangos de Wilcoxon permitió situar las diferencias estadísticamente significativas en varias de las competencias (ver tabla 2). 
Figura 1.

Boxplot de las competencias en función de su relevancia para el alumnado.

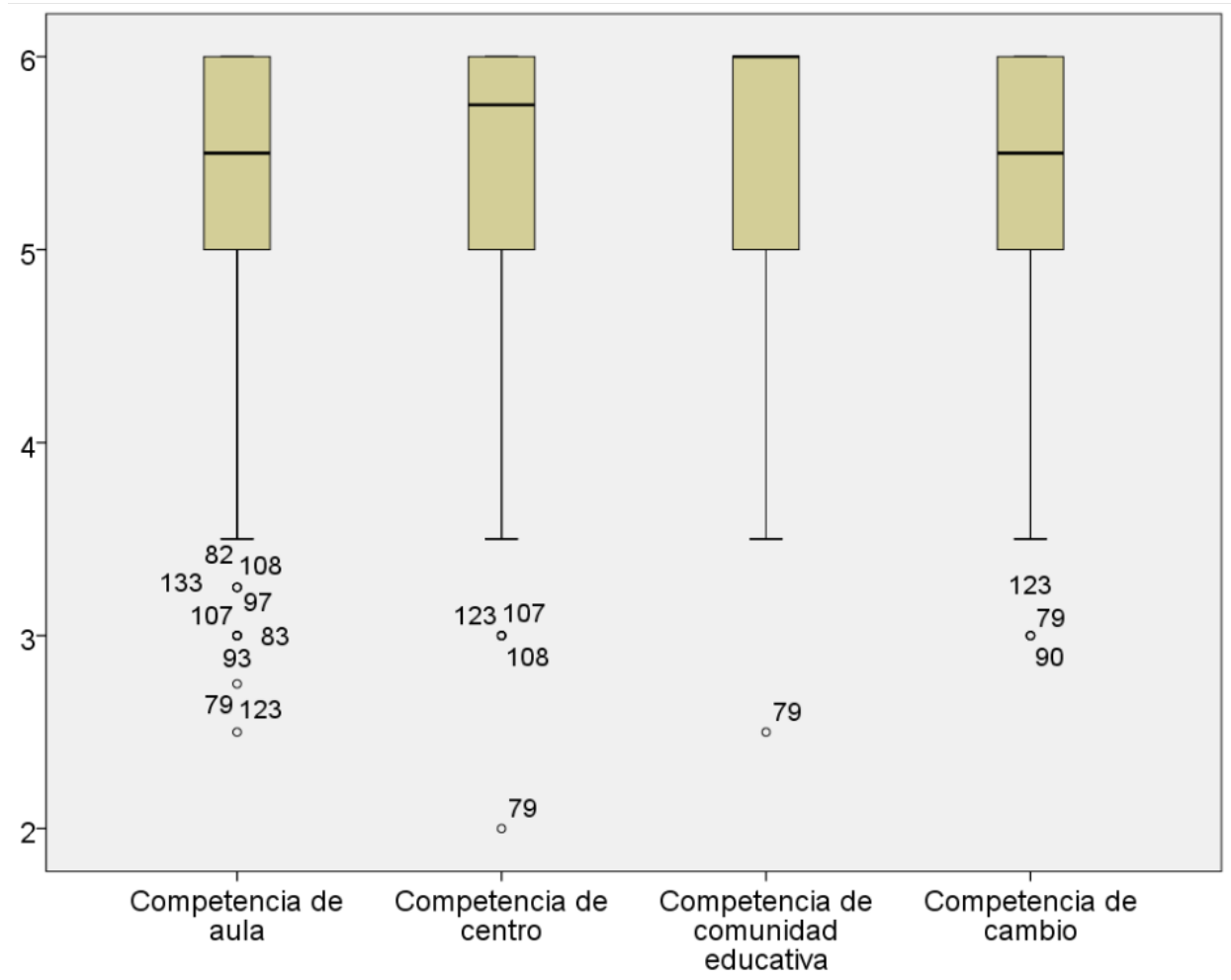

Fuente: elaboración propia

Tabla 2.

Prueba de rangos con signo de Wilcoxon para el análisis de las competencias del modelo

\begin{tabular}{|c|c|c|c|c|}
\hline Competencias & Rangos & $\mathrm{n}$ & Rango promedio & Z \\
\hline \multirow{4}{*}{$\begin{array}{c}\text { Educador/a guía } \\
\text { Miembro de organización }\end{array}$} & Positivos & 46 & 41.83 & \multirow{4}{*}{-1.252} \\
\hline & Norativa & ז & 2001 & \\
\hline & & & & \\
\hline & Empates & 63 & & \\
\hline \multirow{3}{*}{$\begin{array}{c}\text { Educador/a guía } \\
\text { Interlocutor/a y referente }\end{array}$} & Positivos & $60^{a}$ & 45.88 & \multirow{3}{*}{$-3.819 * *$} \\
\hline & Negativos & 26 & 38.02 & \\
\hline & Empates & 58 & & \\
\hline \multirow{3}{*}{$\begin{array}{c}\text { Educador/a guía } \\
\text { Investigador/a e innovador/a }\end{array}$} & Positivos & 48 & 44.22 & \multirow{3}{*}{-1.732} \\
\hline & Negativos & 35 & 38.96 & \\
\hline & Empates & 61 & & \\
\hline \multirow{3}{*}{$\begin{array}{l}\text { Miembro de organización } \\
\text { Interlocutor/a y referente }\end{array}$} & Positivos & 44 & 34.98 & \multirow{3}{*}{$-3.377^{* *}$} \\
\hline & Negativos & $20^{\mathrm{b}}$ & 27.05 & \\
\hline & Empates & 80 & & \\
\hline
\end{tabular}




$\begin{array}{ccccc}\text { Miembro de organización } & \text { Positivos } & 35 & 39.59 & -636 \\ \text { Investigador/a e innovador/a } & \text { Negativos } & 36 & 32.51 & \\ & \text { Empates } & 73 & 31.27 & -2.181^{*} \\ \text { Interlocutor/a y referente } & \text { Positivos } & 18 & 38.32 & \\ \text { Investigador/a e innovador/a } & \text { Negativos } & 28^{\mathrm{c}} & & \end{array}$

${ }^{*} p<.05,{ }^{* *} p<.01$

Nota: basado en rangos negativos donde a. Interlocutor/a y referente > Educador/a guía; b. Interlocutor/a y referente > Miembro de una organización; c. Interlocutor/a y referente > Investigador/a e innovador/a.

Como se puede observar en la tabla 2, sólo se encontraron diferencias estadísticamente significativas a favor de la competencia interlocutor/a y referente, con respecto a educador/a guía $(p<.001)$, miembro de una organización $(p<.001)$ e investigador/a e innovador/a $(p<$ $.001)$.

Para ofrecer una mayor profundidad en este análisis, atendiendo a los componentes de cada una de las cuatro competencias evaluadas, se presenta un análisis descriptivo de las subcompetencias establecidas en el modelo (tabla 3).

Tabla 3.

Estadísticos descriptivos de las subcompetencias del modelo

\begin{tabular}{|c|c|c|c|c|c|c|c|}
\hline Competencia & $\begin{array}{l}\text { Subcompetenci } \\
\text { a }\end{array}$ & $n$ & Md & $P_{25}$ & $\mathrm{P}_{75}$ & Min. & Máx. \\
\hline \multirow{4}{*}{ Educador/a guía } & $\begin{array}{l}\text { Programación, } \\
\text { seguimiento y } \\
\text { evaluación }\end{array}$ & 144 & 5.00 & 4.00 & 6.00 & 1.00 & 6.00 \\
\hline & $\begin{array}{l}\text { Didácticas } \\
\text { específicas, } \\
\text { metodologías, } \\
\text { TAC y alfin }\end{array}$ & 143 & 5.00 & 4.00 & 6.00 & 3.00 & 6.00 \\
\hline & $\begin{array}{l}\text { Orientación } \\
\text { pedagógica y } \\
\text { atención a la } \\
\text { diversidad }\end{array}$ & 144 & 6.00 & 5.00 & 6.00 & 2.00 & 6.00 \\
\hline & $\begin{array}{l}\text { Gestión de los } \\
\text { espacios, } \\
\text { recursos y } \\
\text { materiales de } \\
\text { aprendizaje }\end{array}$ & 137 & 6.00 & 5.00 & 6.00 & 2.00 & 6.00 \\
\hline $\begin{array}{l}\text { Miembro de } \\
\text { organización }\end{array}$ & $\begin{array}{l}\text { Acción tutorial } \\
\text { y orientación } \\
\text { pedagógica, }\end{array}$ & 144 & 5.50 & 4.50 & 6.00 & 2.00 & 6.00 \\
\hline
\end{tabular}




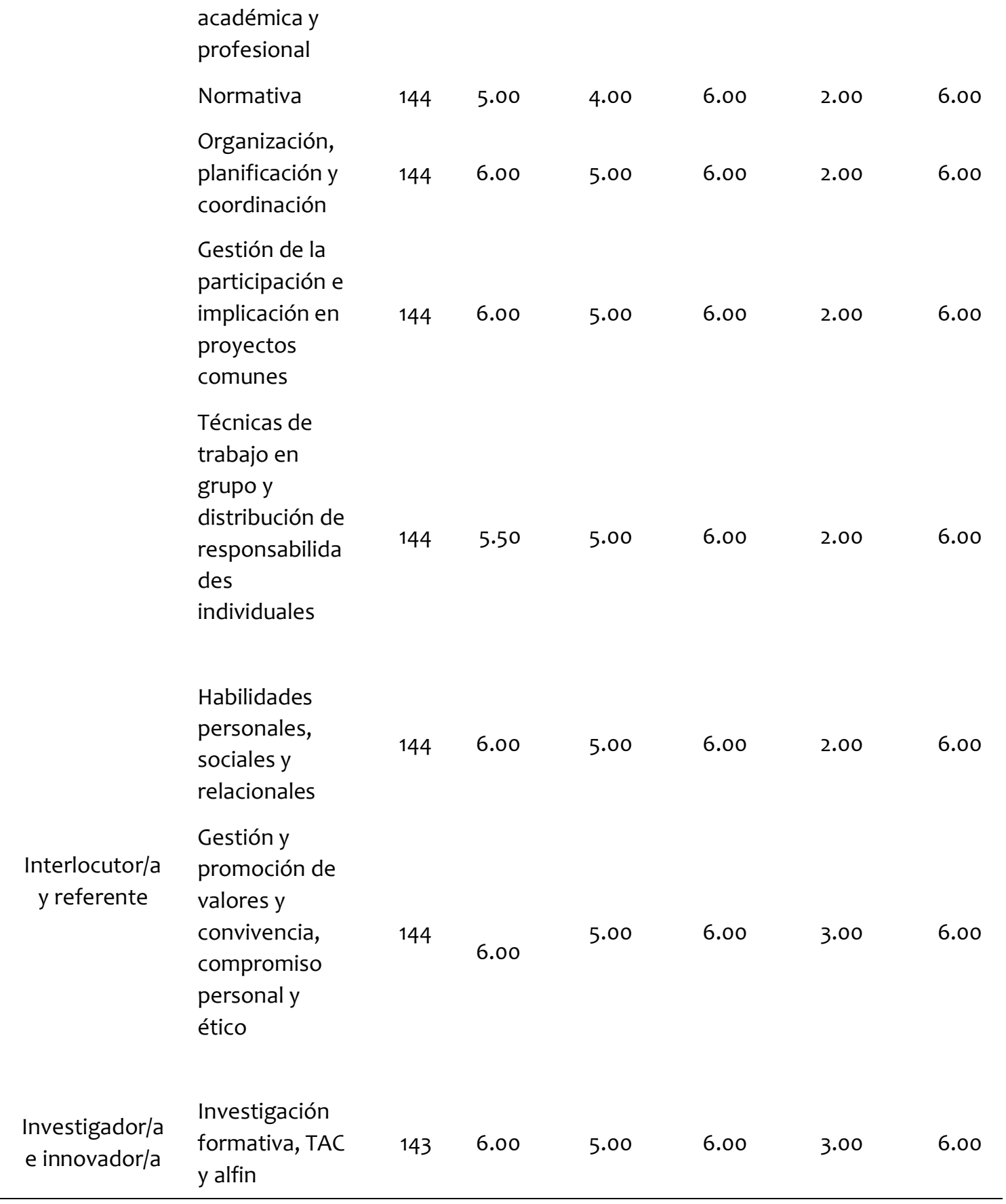

En la tabla 3 se puede observar que el alumnado otorga una alta relevancia a las subcompetencias que integran su perfil profesional, oscilando entre 4 y 6 (para una escala de 6 puntos).

Entre las competencias con mejores valoraciones, destacó la de interlocutor/a y referente puesto que obtuvo en sus dos subcompetencias las medianas más altas $(M d=6)$, destacando la "Gestión y promoción de valores y convivencia, compromiso personal y ético" en la que, además, se observó un posicionamiento de las respuestas entorno a los valores $P_{25}=5$ y $P_{75}$ $=6$, siendo en todo caso, valores superiores al límite 3 .

En la competencia educador/a guía, el alumnado ofreció mayor relevancia a la "Gestión de los espacios, recursos y materiales de aprendizaje" y la "Orientación pedagógica y atención a la 
diversidad", en ambos casos con una $M d=6$, y valores que se posicionaron en torno a $\mathrm{P}_{25}=5$ y $\mathrm{P}_{75}=6$ aunque, en este caso, el límite inferior se situó en el punto 2 .

Los mismos valores se encontraron en "Gestión de la participación e implicación en proyectos comunes" ( $\left.M d=6 ; \mathrm{P}_{25}=5 ; \mathrm{P}_{75}=6\right) \mathrm{y}$, medio punto por debajo, "Técnicas de trabajo en grupo y distribución de responsabilidades individuales" ( $\left.M d=5.5 ; \mathrm{P}_{25}=5 ; \mathrm{P}_{75}=6\right)$, ambas contempladas en la competencia Miembros de una organización.

Finalmente, la competencia investigador/a e innovador/a, con una única subcompetencia, obtuvo una valoración elevada dentro de la escala de valores, y también en relación con los de las otras subcompetencias $\left(M d=6 ; \mathrm{P}_{25}=5 ; \mathrm{P}_{75}=6\right)$.

Se confirmó, a través de la prueba de Friedman $\left(\chi^{2}{ }_{11}=145.70 ; p=.001\right)$, la existencia de diferencias estadísticamente significativas en la valoración de las subcompetencias. Adicionalmente, la prueba de rangos de Wilcoxon permitió ubicar dichas diferencias entre cada una de las competencias (tabla 4).

Tabla 4.

Prueba de rangos con signo de Wilcoxon para el análisis de las subcompetencias (SC) del modelo

\begin{tabular}{|c|c|c|c|c|}
\hline $\mathrm{SC}_{1}$ & $\mathrm{SC} 2$ & $N$ & $\begin{array}{c}\text { Rango } \\
\text { Promedio }\end{array}$ & Z \\
\hline & $\begin{array}{l}\text { Didácticas específicas, metodologías, } \\
\text { TAC y alfin }\end{array}$ & $55^{\mathrm{a}}$ & 50.07 & $-2.43^{*}$ \\
\hline & $\begin{array}{l}\text { Orientación pedagógica y atención a la } \\
\text { diversidad }\end{array}$ & $84^{a}$ & 48.89 & $-7.15^{* *}$ \\
\hline & $\begin{array}{l}\text { Gestión de los espacios, recursos y } \\
\text { materiales de aprendizaje }\end{array}$ & $76^{\mathrm{a}}$ & 49.03 & $-5.69 * *$ \\
\hline & $\begin{array}{l}\text { Acción tutorial y orientación } \\
\text { pedagógica, académica y profesional }\end{array}$ & $71^{\mathrm{a}}$ & 49.54 & $-4 \cdot 90 * *$ \\
\hline & $\begin{array}{l}\text { Organización, planificación y } \\
\text { coordinación }\end{array}$ & $69^{a}$ & 49.33 & $-5.24^{* *}$ \\
\hline \multirow[t]{5}{*}{ PSE } & $\begin{array}{l}\text { Gestión de la participación e } \\
\text { implicación en proyectos comunes }\end{array}$ & $73^{\mathrm{a}}$ & 53.52 & $-4 \cdot 79^{* *}$ \\
\hline & $\begin{array}{l}\text { Técnicas de trabajo en grupo y } \\
\text { distribución de responsabilidades } \\
\text { individuales }\end{array}$ & $72^{\mathrm{a}}$ & 65.77 & $-5.06 * *$ \\
\hline & $\begin{array}{l}\text { Habilidades personales, sociales y } \\
\text { relacionales }\end{array}$ & $78^{\mathrm{a}}$ & 53.61 & $-6.01 * *$ \\
\hline & $\begin{array}{l}\text { Gestión y promoción de valores y } \\
\text { convivencia, compromiso personal y } \\
\text { ética }\end{array}$ & $84^{\mathrm{a}}$ & 49.13 & $-7.20 * *$ \\
\hline & Investigación formativa, TAC y alfin & $69^{a}$ & 52.02 & $-4 \cdot 16 * *$ \\
\hline DID & $\begin{array}{l}\text { Orientación pedagógica y atención a la } \\
\text { diversidad }\end{array}$ & $59^{a}$ & 34.47 & $-6.18 * *$ \\
\hline
\end{tabular}




\begin{tabular}{|c|c|c|c|c|}
\hline & $\begin{array}{l}\text { Gestión de los espacios, recursos y } \\
\text { materiales de aprendizaje }\end{array}$ & $50^{a}$ & $35 \cdot 31$ & $-4 \cdot 42^{* *}$ \\
\hline & $\begin{array}{l}\text { Acción tutorial y orientación } \\
\text { pedagógica, académica y profesional }\end{array}$ & $53^{a}$ & 44.32 & $-3 \cdot 30 * *$ \\
\hline & $\begin{array}{l}\text { Organización, planificación y } \\
\text { coordinación }\end{array}$ & $47^{\mathrm{a}}$ & 39.41 & $-4.01 * *$ \\
\hline & $\begin{array}{l}\text { Gestión de la participación e } \\
\text { implicación en proyectos comunes }\end{array}$ & $50^{a}$ & 38.08 & $-3 \cdot 48 * *$ \\
\hline & $\begin{array}{l}\text { Técnicas de trabajo en grupo y } \\
\text { distribución de responsabilidades } \\
\text { individuales }\end{array}$ & $58^{a}$ & 52.28 & $-4.01 * *$ \\
\hline & $\begin{array}{l}\text { Habilidades personales, sociales y } \\
\text { relacionales }\end{array}$ & $55^{\mathrm{a}}$ & 37.05 & $-5.16 * *$ \\
\hline & $\begin{array}{l}\text { Gestión y promoción de valores y } \\
\text { convivencia, compromiso personal y } \\
\text { ético }\end{array}$ & $66^{\mathrm{a}}$ & 43.92 & $-6.25 * *$ \\
\hline & Investigación formativa, TAC y alfin & $47^{\mathrm{a}}$ & 41.12 & $-3.03^{*}$ \\
\hline \multirow{4}{*}{ OPAD } & $\begin{array}{l}\text { Acción tutorial y orientación } \\
\text { pedagógica, académica y profesional }\end{array}$ & $25^{\mathrm{b}}$ & 21.60 & $-3.96 * *$ \\
\hline & Normativa & $55^{b}$ & 35.86 & $-4.67 * *$ \\
\hline & $\begin{array}{l}\text { Organización, planificación y } \\
\text { coordinación }\end{array}$ & $39^{b}$ & 24.45 & $-2.50 *$ \\
\hline & Investigación formativa, TAC y alfin & $40^{a}$ & 31.20 & $-2.23^{*}$ \\
\hline \multirow{3}{*}{ GERM } & $\begin{array}{l}\text { Acción tutorial y orientación } \\
\text { pedagógica, académica y profesional }\end{array}$ & $49^{b}$ & 34.91 & $-2.85 * *$ \\
\hline & Normativa & $48^{b}$ & 37.71 & $-3.73^{* *}$ \\
\hline & $\begin{array}{l}\text { Gestión y promoción de valores y } \\
\text { convivencia, compromiso personal y } \\
\text { ético }\end{array}$ & $37^{\mathrm{a}}$ & 29.55 & $-2.77^{*}$ \\
\hline \multirow{3}{*}{ ATOP } & Normativa & $53^{b}$ & 49.27 & $-2.98 * *$ \\
\hline & $\begin{array}{l}\text { Habilidades personales, sociales y } \\
\text { relacionales }\end{array}$ & $54^{a}$ & 39.06 & $-2.65^{* *}$ \\
\hline & $\begin{array}{l}\text { Gestión y promoción de valores y } \\
\text { convivencia, compromiso personal y } \\
\text { ético }\end{array}$ & $60^{a}$ & 38.19 & $-4.99 * *$ \\
\hline \multirow{2}{*}{ NOR } & $\begin{array}{l}\text { Organización, planificación y } \\
\text { coordinación }\end{array}$ & $43^{a}$ & 32.93 & $-3.79 * *$ \\
\hline & $\begin{array}{l}\text { Gestión de la participación e } \\
\text { implicación en proyectos comunes }\end{array}$ & $51^{a}$ & 38.76 & $-3.27 * *$ \\
\hline
\end{tabular}




\begin{tabular}{|c|c|c|c|c|}
\hline & $\begin{array}{l}\text { Técnicas de trabajo en grupo y } \\
\text { distribución de responsabilidades } \\
\text { individuales }\end{array}$ & $55^{\mathrm{a}}$ & 46.19 & $-4 \cdot 18 * *$ \\
\hline & $\begin{array}{l}\text { Habilidades personales, sociales y } \\
\text { relacionales }\end{array}$ & $49^{a}$ & 36.27 & $-4.73^{* *}$ \\
\hline & $\begin{array}{l}\text { Gestión y promoción de valores y } \\
\text { convivencia, compromiso personal y } \\
\text { ético }\end{array}$ & $59^{a}$ & 39.39 & $-5.74 * *$ \\
\hline & Investigación formativa, TAC y alfin & $43^{a}$ & 36.34 & $-3.29 * *$ \\
\hline \multirow[b]{2}{*}{ OPC } & $\begin{array}{l}\text { Habilidades personales, sociales y } \\
\text { relacionales }\end{array}$ & $19^{a}$ & 25.68 & $-1.95^{*}$ \\
\hline & $\begin{array}{l}\text { Gestión y promoción de valores y } \\
\text { convivencia, compromiso personal y } \\
\text { ético }\end{array}$ & $10^{a}$ & 22.30 & $-4 \cdot 91 * *$ \\
\hline GPI & $\begin{array}{l}\text { Gestión y promoción de valores y } \\
\text { convivencia, compromiso personal y } \\
\text { ético }\end{array}$ & $41^{\mathrm{b}}$ & 29.62 & $-2.74^{* *}$ \\
\hline TTG & $\begin{array}{l}\text { Gestión y promoción de valores y } \\
\text { convivencia, compromiso personal y } \\
\text { ético }\end{array}$ & $48^{a}$ & 32.90 & $-3.69 * *$ \\
\hline \multirow[t]{2}{*}{ HPSR } & $\begin{array}{l}\text { Gestión y promoción de valores y } \\
\text { convivencia, compromiso personal y } \\
\text { ético }\end{array}$ & $16^{\mathrm{a}}$ & 19.91 & $-2.48 * *$ \\
\hline & Investigación formativa, TAC y alfin & $19^{b}$ & 23.18 & $-2.40 *$ \\
\hline GPVC & Investigación formativa, TAC y alfin & $14^{b}$ & 24.25 & $-4.00 * *$ \\
\hline
\end{tabular}

Nota: Se presentan únicamente los resultados estadísticamente significativos. * $p<.05,{ }^{* *} p<.01$

a. basado en rangos positivos donde Subcompetencia $1<$ Subcompetencia 2.

b. basado en rangos negativos donde Subcompetencia $2<$ Subcompetencia 1

Siglas: PSE - Programación, seguimiento y evaluación, DID - Didácticas específicas, metodologías, TAC y alfin, OPAD - Orientación pedagógica y atención a la diversidad, GERM - Gestión de espacios, recursos y materiales de aprendizaje, ATOP - Acción tutorial y orientación pedagógica, académica y profesional, NOR - Normativa, OPC - Organización, planificación, coordinación, GPI - Gestión de participación e implicación en proyectos comunes, TTG - Técnicas de trabajo en grupo y distribución de responsabilidades individuales, HPSR - Habilidades personales, sociales y relacionales, GPVC Gestión y promoción de valores y convivencia.

Hubo diferencias estadísticamente significativas entre varias de las subcompetencias del Modelo (ver tabla 4). Destacó la escasa valoración del alumnado hacia la relevancia que tiene formarse en "programación, seguimiento y evaluación" con respecto al resto de subcompetencias (a excepción de "Normativa"). Seguidamente, la subcompetencia "Didácticas específicas, metodologías, TAC y alfin" obtuvo puntuaciones significativamente bajas en cuanto a relevancia frente a las demás subcompetencia, excepto la citada "programación, seguimiento y evaluación” y "normativa". 
En el lado opuesto, encontramos que las subcompetencias significativamente mejor valoradas por su relevancia fueron las vinculadas a la capacidad de gestión y desarrollo personal en entorno de aula, centro y comunidad educativa; esto es, la "gestión de los espacios, recursos y materiales de aprendizaje", como competencia de aula, la "gestión de la participación e implicación en proyectos comunes", como competencia de centro, y las "habilidades personales, sociales y relacionales", entre las competencias comunidad educativa.

\subsection{Correlaciones entre competencias y subcompetencias del Modelo Competencial}

Finalmente, se estudió la posible relación entre la relevancia concedida a las competencias y subcompetencias evaluadas mediante la prueba Rho de Spearman (ver tablas 5 y 6 ).

Tabla 5.

Correlaciones entre las competencias del modelo

\begin{tabular}{|c|c|c|c|c|}
\hline & $\begin{array}{c}\text { Educador/a } \\
\text { guía }\end{array}$ & $\begin{array}{l}\text { Miembro de } \\
\text { organización }\end{array}$ & $\begin{array}{l}\text { Interlocutor/a y } \\
\text { referente }\end{array}$ & $\begin{array}{c}\text { Investigador/a e } \\
\text { innovador/a }\end{array}$ \\
\hline Educador/a guía & 1 & $.663^{*}$ & $.666^{*}$ & $.591^{*}$ \\
\hline $\begin{array}{l}\text { Miembro de } \\
\text { organización }\end{array}$ & & 1 & $.772^{*}$ & $.692^{*}$ \\
\hline $\begin{array}{l}\text { Interlocutor/a y } \\
\text { referente }\end{array}$ & & & 1 & $.655^{* *}$ \\
\hline $\begin{array}{c}\text { Investigador/a e } \\
\text { innovador/a }\end{array}$ & & & & 1 \\
\hline
\end{tabular}

Nota: $* p=.01$

La tabla 5 muestra correlaciones estadísticamente significativas entre las cuatro competencias estudiadas, siendo destacable entre miembro de una organización e interlocutor/a y guía por su carácter positivo y fuerte $(r<.772)$. En el resto de los casos, se observaron correlaciones elevadas y positivas, todas ellas superiores al $50 \%$ de relación.

Tabla 6.

Correlaciones entre las subcompetencias del modelo

\begin{tabular}{|c|c|c|c|c|c|c|c|c|c|c|c|c|}
\hline & PSE & DID & OPAD & GERM & ATOP & NOR & OPC & GPI & TTG & HPSR & GPVC & IFTAC \\
\hline PSE & 1 & $.56^{* *}$ & $.62^{* *}$ & $.47^{* *}$ & $.48^{* *}$ & $.34^{* *}$ & $.39 * *$ & $.31^{* *}$ & $.18^{*}$ & $.36 * *$ & $.40^{* *}$ & $.31^{* *}$ \\
\hline DID & & 1 & $.67^{* *}$ & $.52^{* *}$ & $.64^{* *}$ & $.41^{* *}$ & $.55^{* *}$ & $.51^{* *}$ & $.43^{* *}$ & $.56^{* *}$ & $.50^{* *}$ & $.50^{* * *}$ \\
\hline OPAD & & & 1 & $.730^{* *}$ & $.71 * *$ & $.43^{* *}$ & $.64^{* *}$ & $.58^{* *}$ & $.48^{* *}$ & $.62^{* *}$ & $.69^{* *}$ & $.48^{* *}$ \\
\hline GERM & & & & 1 & $.74^{* *}$ & $.39 * *$ & $.62^{* *}$ & $.54^{* *}$ & $.51^{* *}$ & $.52^{* *}$ & $.55 * *$ & $.35^{* *}$ \\
\hline ATOP & & & & & 1 & $56 * *$ & $.78^{* *}$ & $.66^{* *}$ & $.64^{* *}$ & $.61^{* *}$ & $.63^{* *}$ & $.55^{* *}$ \\
\hline NOR & & & & & & 1 & $.60 * *$ & $.41^{* * *}$ & $.55^{* *}$ & $.46^{* *}$ & $.46^{* *}$ & $.54^{* *}$ \\
\hline OPC & & & & & & & 1 & $.74^{* *}$ & $.67^{* *}$ & $.62^{* *}$ & $.73^{* *}$ & $.52 * *$ \\
\hline GPI & & & & & & & & 1 & $.59^{* *}$ & $.56^{* *}$ & $.61^{* *}$ & $.53^{* *}$ \\
\hline TाG & & & & & & & & & 1 & $58^{* *}$ & $.63^{* *}$ & $.51 * *$ \\
\hline
\end{tabular}




\begin{abstract}
HPSR
GPVC

IFTAC

$1.69^{* *} \quad .53^{* *}$

$1.50^{* *}$

Nota: ${ }^{*} p=.05, * * p=.01$

Siglas: PSE - Programación, seguimiento y evaluación, DID - Didácticas específicas, metodologías, TAC y alfin, OPAD - Orientación pedagógica y atención a la diversidad, GERM - Gestión de espacios, recursos y materiales de aprendizaje, ATOP - Acción tutorial y orientación pedagógica, académica y profesional, NOR - Normativa, OPC - Organización, planificación, coordinación, GPI - Gestión de participación e implicación en proyectos comunes, TTG - Técnicas de trabajo en grupo y distribución de responsabilidades individuales, HPSR - Habilidades personales, sociales y relacionales, GPVC Gestión y promoción de valores y convivencia, IFTAC - Investigación formativa, TAC y alfin.
\end{abstract}

La tabla 6 evidencia la presencia de correlaciones entre todas las subcompetencias especificadas en el modelo. Dichas correlaciones son, en todos los casos positivas. Entre las correlaciones más elevadas $(r>.75)$ destacó un 78\% de asociación entre "Organización, planificación, coordinación" (competencia de centro) y "Acción tutorial y orientación pedagógica, académica y profesional” (competencia de centro), así como un $75 \%$ entre la primera y "Gestión y promoción de valores y convivencia" (competencia de comunidad educativa). Por el lado opuesto, la asociación con un índice menos elevado de correlación ( $r$ <.30) fue la que se estableció entre "Programación, seguimiento y evaluación" y "Técnicas de trabajo en grupo y distribución de responsabilidades individuales".

\title{
Discusión y conclusiones
}

Los datos obtenidos en este estudio han revelado la importancia que las y los futuros profesionales conceden a su formación integral y, especialmente, a todas aquellas competencias que se relacionan con el compromiso con su profesión, con la formación en valores y para la convivencia, y con el desarrollo de sus habilidades personales y sociales. En este sentido, De Haro et al. (2020) señalan que la formación en competencias del maestro es el resultado de un proceso de aprendizaje inicial, y también continuo, que le capacita para un desempeño adecuado de sus funciones, al tiempo que sienta las bases de su identidad personal, ética, humana y profesional.

De forma específica, ha sido posible extraer varias ideas clave en este estudio en relación con la relevancia otorgada a la formación de maestros en educación infantil bajo el Modelo Competencial de la Rede de Formación de la Xunta de Galicia.

En primer lugar, la competencia de interlocutor/a y referente fue la mejor valorada, evidenciando un mayor peso de la formación en habilidades que favorecen un clima positivo en los centros educativos. Este dato es coherente con estudios recientes en los que se ha obtenido una preponderancia de las competencias vinculadas al componente emocional, comunicativo y/o socioafectivo (Domínguez et al., 2015; Domínguez, González, Medina y Medina, 2017; González-Fernández et al., 2019) y con trabajos que señalan la importancia de las actitudes del docente y su disposición hacia el aprendizaje (Forteza, 2011) como forma de demostrar el compromiso ético con su ejercicio profesional y la activación de sus competencias profesionales (Pérez, 2013). 
En segundo lugar, confirieron gran relevancia a la competencia miembro de una organización. Esta elevada valoración de las competencias de centro, sobre todo del trabajo grupal y distribución de responsabilidades, así como de la gestión de la participación e implicación en proyectos comunes podría ser claro reflejo del modelo de enseñanza universitaria vivenciado en la formación inicial de los/as maestros/as y tan característico del EEES que apuesta por el desarrollo de un aprendizaje eminentemente práctico y por metodologías de enseñanza y de evaluación basadas en el trabajo en grupo (García et al., 2011; Pérez-Ferra, Sierra-Arizmendiarreta y Quijano-López, 2018). Del mismo modo, la importancia que se ofrece en las titulaciones de educación no solo a la formación en orientación, tutoría y acción tutorial, sino también a modelar los aprendizajes por medio de tutorías universitarias y recursos como el Plan de Acción Tutorial pueden haber fomentado los resultados positivos obtenidos en estas subcompetencias (Zabalza, 2009).

También se ha encontrado una elevada correlación entre las competencias del Modelo que revelan la importancia de disponer de un modelo de formación integral, que revierta sobre la actividad en el aula, el centro, la comunidad educativa y como eje de cambio social (GonzálezFernández et al., 2019). Destaca, sobre todo, la elevada correlación entre interlocutor/a y referente y miembro de una organización, que refleja la importancia de la ética, los valores y el espíritu colaborador para la atención a la diversidad y la orientación educativa (Díez, Rodríguez y Mallo, 2014) y evidencia que la actitud y la reflexión sobre la práctica aporta opciones de mejora. A la luz de unos resultados que evidencian una mayor relevancia de las competencias relacionadas con las habilidades personales y sociales, el trabajo en equipo, la coordinación y la corresponsabilidad, se deduce que la adquisición de competencias implica también una formación en actitudes críticas y creativas hacia la búsqueda continua de formación, indagación y mejora (Martínez, 2018).

El reconocimiento y valoración positiva que se otorgan a la formación inicial repercutirá, no solo en la capacitación que se adquiere, sino también en la predisposición y actitud hacia la puesta en práctica de los aprendizajes adquiridos una vez se acceda al mercado laboral. Ello sitúa al espacio universitario como caldo de cultivo ideal para la formación, no solo de las destrezas que capacitan para la profesión, sino de aquellas habilidades que conviertan a los/as docentes en ciudadanos/as con capacidad de opinar, de aprender, de adaptarse, con espíritu crítico y con creatividad (Pérez y Castaño, 2016), siendo capaces de realizar una continua reflexión en la acción que motive la creación de nuevo conocimiento y de investigar sobre su práctica (Sabariego, Cano, Gros y Piqué, 2020).

En definitiva, este trabajo permite visibilizar dos cuestiones de amplia trascendencia en el Espacio Europeo de Educación Superior: la primera es la importancia de la evaluación de las percepciones del profesorado en formación, pues ello contribuye a que el profesorado universitario pueda facilitarles un aprendizaje adaptado a sus necesidades y que posibilite que los/as estudiantes tengan una visión más realista de su profesión y de sí mismos como futuros educadores (Asensio Muñoz y Ruíz de Miguel, 2017). La segunda cuestión redunda en reforzar, la importancia de la formación continua pues, como reconoce Forteza (2011) la mejor forma de avanzar, de aprender y de cambiar concepciones es mediante la formación permanente. 


\section{Referencias}

Agencia Nacional de Evaluación de la Calidad y Acreditación (2004). Libro blanco título de grado en magisterio (Vol. 1). Recuperado de http://www.aneca.es/var/media/150404/libroblanco_jun05_magisterio1.pdf

Asensio Muñoz, l., \& Ruiz de Miguel, C. (2017). Medida y evaluación de las creencias sobre la profesión de los maestros en formación. Revista Electrónica Interuniversitaria de Formación del Profesorado, 20(3), 79-91. http://dx.doi.org/10.6018/reifop.20.3.265231

Bas-Peña, E., Ferre-Jaén, E., \& Maurandi-López, A. (2020). Validación de un cuestionario mediante un modelo de ecuaciones estructurales para conocer percepciones del alumnado sobre competencias profesionales y habilidades sociales en los grados de educación. Revista Electrónica Educare, 24 (3), 1-20. doi: http://doi.org/10.15359/ree.243.1

Cejudo, J., Díaz, M. V., Losada, M. L., \& Pérez-González, J. C. (2016). Necesidades de formación de maestros de infantil y primaria en atención a la diversidad. Bordón. Revista De Pedagogía, 68(3), 23-39. doi: http://doi.org/10.13042/Bordon.2016.68402

Consellería de Cultura, Educación y Ordenación Universitaria (2015). Las competencias profesionales docentes. Modelo competencial de la Red de Formación de Profesorado. Galicia: Xunta de Galicia, Centro Autonómico de Formación e Innovación.

Consellería de Cultura, Educación y Ordenación Universitaria (2017). Mellora da formación docente en Galicia: Competencias profesionais. Galicia: Xunta de Galicia, Universidade de Santiago, Universidade de Vigo e Universidade da Coruña.

Cuadrado, I., Monroy, F.A., \& Montaño, A. (2011). Características propias de los estilos de aprendizaje de los estudiantes de maestro en educación infantil. International Journal of Developmental and Educational Psychology, 1(3), 217-226. Recuperado de https://www.redalyc.org/pdf/3498/349832330022.pdf

Cuberos, M., Santamaría, A., Prados. M.M., \& Arias, S. (2019). Concepciones del aprendizaje de estudiantes en proceso de formación como docentes. Profesorado. Revista de Currículum y Formación del Profesorado, 23(3), 453-471. doi: https://doi.org/10.30827/profesorado.v23i3.11238

De Haro, R., Arnáiz-Sánchez, P. \& Núñez de Perdomo, C.R. (2020). Teacher competences in Early Childhood Education and inclusive education: Design and validation of a questionnaire. Revista Electrónica Interuniversitaria de Formación del Profesorado, 23(1), 1-20. https://doi.org/10.6018/reifop.407111

Díez, E.J., Rodríguez, J.R., \& Mallo, B. (2014). La formación inicial del profesorado en el "habitus" capitalista. Cuadernos de pedagogía, 445, 75-78. Recuperado de https://dialnet-unirioja-es.accedys.udc.es/servlet/articulo?codigo $=4696387$

Domínguez, M.C., González, R., Medina, M.C., \& Medina, A. (2015). Formación inicial del profesorado de Educación Infantil: claves para el diseño innovador de planes de estudio. ENSAYOS, Revista de la Facultad de Educación de Albacete, 30(2), 227-245. Recuperado de http://www.revista.uclm.es/index.php/ensayos

Domínguez, M.C., González, R., Medina, M., \& Medina, A. (2017). Desarrollo de Competencias Básicas en la formación Inicial del Profesorado de Educación Infantil, Pedagogía PIU' Didattica, 3(1), 1-10. Recuperado de http://rivistedigitali.erickson.it/pedagogia-piudidattica/archivio/vol-3-n-1/ 
Feisal, J. (2015). La armonización en el Espacio Europeo de Educación Superior: estado de la cuestión. Journal of Supranational Policies of Education, 3, 264-282. Recuperado de https://repositorio.uam.es/bitstream/handle/10486/667468/JOSPOE_3_16.pdf?sequen ce=1\&isAllowed $=y$

Fernández, C., Hervás, M., Aparicio, M., Polo, M.T., \& Tallón, S. (2019). Formación en competencias del alumnado de educación en la atención a la diversidad: diferencias según la titulación. International Journal of Developmental and Educational Psychology, 1 (2), 497-502. doi: https://doi.org/10.17060/ijodaep.2019.n1.v3.1526

Forteza, D. (2011). Algunas claves para repensar la formación del profesorado sobre la base de la inclusión. Revista Interuniversitaria de Formación del Profesorado, 25(1), 127-144. Recuperado de https://www.redalyc.org/pdf/274/27419147008.pdf

Gairín, J. (2011). Formación de profesores basada en competencias. Bordón. Revista Española de Pedagogía, 63(1), 93-108. Recuperado de https://recyt.fecyt.es/index.php/BORDON/article/view/28907

García, J., de Caso, A., I Pacheco, D., Robledo, P., Álvarez, L., García-Martín, J., \& GarcíaMartín, E. (2011). Innovación en el EEES con metodologías activas. International Journal of Developmental And Educational Psychology, 3(1), 365-372. Recuperado de http://infad.eu/RevistalNFAD/2011/n1/volumen3/INFAD_010323_365-372.pdf

Gil, P., Gómez, M. I., \& Rodríguez, M. A. (2013). Las competencias y el modelo de formación de los maestros y maestras de educación infantil. Estudio de caso. En E. Nieto López, A.I. Callejas Albiñana, \& O. Jerez García (Coords.), Las competencias básicas. Competencias profesionales del docente (pp. 258-275). Recuperado de http://publicaciones.dipucr.es/tripascompetenciasdocente.pdf

González-Fernández, R., Zabalza-Cerdeiriña, M.A., Medina-Domínguez, M., \& Medina-Rivilla, A. (2019). Modelo de formación inicial del profesorado de Educación Infantil: Competencias y creencias para su capacitación. Formación Universitaria, 12(2), 83-96. doi: http://doi.org/10.4067/S0718-50062019000200083

Latorre, M., Aravena, P., Milos, P., \& García, M. (2010). Competencias habilitantes: un aporte para el reforzamiento de las trayectorias formativas universitarias. Calidad en la educación, 33, 275-301. Recuperado de https://dialnet.unirioja.es/servlet/articulo?codigo=3732194

López, A.B., González, I., \& De León, C. (2014). Perfil de un buen docente. Aplicación de un protocolo de evaluación de las competencias del profesorado universitario. Revista Electrónica Interuniversitaria de Formación del Profesorado, 17(1), 133-148. doi: http://dx.doi.org/10.6018/reifop.17.1.190531

Martínez, A. (2018). Percepciones sobre las competencias de formación básica en educación infantil. Aula de Encuentro. Revista de investigación y comunicación de experiencias educativas, 20(1), 54-73. doi: https://doi.org/10.17561/ae.v20i1.3

Muñoz-Cantero, J.M., Rebollo, N. y Espiñeira, E. (2014). Percepción de competencias en el EEES: análisis en el Grado de Educación Primaria. Revista Electrónica Interuniversitaria $\begin{array}{llll}\text { de Formación del Profesorado, 17(3), } & \text { 123-139 }\end{array}$ http://dx.doi.org/10.6018/reifop.17.3.204091

Orden ECl/3854/2007, de 27 de diciembre, por la que se establecen los requisitos para la verificación de los títulos universitarios oficiales que habiliten para el ejercicio de la profesión de Maestro en Educación Infantil. BOE núm. 312, de 29 de diciembre, pp. 53735-53738. 
Pérez, M. (2013). La actitud indagadora del profesor: un proceso para desarrollar competencias en los docentes. Profesorado. Revista de Currículum y Formación del Profesorado, 17(3), 57-72. Recuperado de https://www.ugr.es/ recfpro/rev173ART3.pdf

Pérez, S. y Castaño, R. (2016). Funciones de la Universidad en el siglo XXI: humanística, básica e integral. Revista Electrónica Interuniversitaria de Formación del Profesorado, 19(1), 191-199. http://dx.doi.org/10.6018/reifop.19.1.202451

Pérez-Ferra, M., Sierra-Arizmendiarreta, B., \& Quijano-López, R. (2018). Percepción y creencias del alumnado de primero de los títulos de grado de educación infantil y primaria, en Jaén y Oviedo acerca de la competencia 'trabajo en equipo'. Profesorado. Revista de Currículum y Formación del Profesorado, 22(3), 9-29. doi: https://doi.org/10.30827/profesorado.v22i3.7988

Ramírez, A. (2015). La formación del profesorado de Educación Primaria ante las competencias básicas. Revista Electrónica Interuniversitaria de Formación del Profesorado, 18(3), 199-214. http://dx.doi.org/10.6018/reifop.18.3.193811

Rosales, C. (2013). Competencias específicas curriculares que ha de adquirir el estudiante del título de grado de maestro. Profesorado. Revista de Currículum y Formación del Profesorado, 17(3), 73-90. Recuperado de https://recyt.fecyt.es/index.php/profesorado/article/view/41766/23812

Sabariego, M., Cano, A.N., Gros, B., \& Piqué, B. (2020). Competencia investigadora e investigación formativa en la formación inicial del docente. Contextos educativos, 26, 239-259. doi: http://doi.org/10.18172/con.4326

Tejada, J., \& Ruiz, C. (2013). Significación del prácticum en la adquisición de competencias profesionales que permiten la transferencia de conocimiento a ámbitos propios de la acción docente. Profesorado. Revista de Currículum y Formación del Profesorado, 17 (3), 91-110. Recuperado de https://recyt.fecyt.es/index.php/profesorado/article/view/41974

Zabalza, M. A. (2009). Ser profesor universitario hoy. Revista La cuestión universitaria, 13(5), 68-80. Recuperado de http://polired.upm.es/index.php/lacuestionuniversitaria/article/view/3338/3403 